\title{
Sportmedizin und Rehabilitation
}

Sport gehört-wer möchte dies bezweifeln - zu den in der breiten Öffentlichkeit am häufigsten diskutierten Themen. Ursprünglich nur in eigens hiefür bestimmten Zeitschriften publiziert und für einen beschränkten Leserkreis bestimmt, dürfte sich heute keine renommierte Tageszeitung den Verzicht auf eine die Sportgeschehnisse kommentierende Beilage leisten.

Sport besetzt aber auch - mindestens in der westlichen Welt, wo die Befriedigung der Überlebensbedürfnisse für die Mehrzahl der Bevölkerung gesichert ist - einen wachsenden Teil der Freizeit, die sich überdies auf Kosten einer schrumpfenden Arbeitszeit immer weiter ausdehnt. Auch der „Seniorensport" gehört längst nicht mehr zu den Neologismen unserer Umgangssprache, sondern wird angesichts der immer weiter fortschrei- tenden Überalterung und dank seinen unbestritten positiven Seiten programmatisch weiter verbreitet.

Sportverletzungen und Sportschäden sind die zwangsläufigen Begleiterscheinungen aller Sportarten. Ihre Häufigkeit hat nicht nur für das betroffene Individuum einschneidende Bedeutung, sie belasten überdies in bedenklicher Weise die abnehmenden finanziellen Ressourcen unserer Sozialstaaten. Da die Mehrzahl dieser Sportverletzungen und -schäden den Bewegungsapparat betreffen, ist der Orthopäde als Arzt mit ihrer Behandlung in allererster Linie konfrontiert. Ein Blick in die orthopädisch-chirurgische Literatur vermag uns mühelos zu überzeugen, daß es an Artikeln über die therapeutischen Maßnahmen von Sportverletzungen und-schäden nicht mangelt. Sind wir aber auch genügend vertraut mit den Grundlagen der Prophylaxe oder mit den durch Krankheit und Operation verursachten strukturellen Verän- derungen des Bewegungsapparats und deren Auswirkungen auf unsere Rehabilitationsmaßnahmen?

In der bestimmten Annahme, daß neuere Forschungsergebnisse der Sportmedizin den wenigsten Lesern unserer Zeitschrift bekannt sein dürften, wurde diese Nummer des Orthopäden in enger Zusammenarbeit mit international anerkannten Fachleuten konzipiert. Die Umsetzung des Inhalts in die Praxis heisst unsere Verordnungen von Rehabilitationsmaßnahmen neu zu überdenken, bedeutet aber auch Hilfeleistung bei der sachkundigen Beratung von Gesunden und Kranken unseres Fachgebiets hinsichtlich der Möglichkeiten und Grenzen einer gesunden körperlichen Aktivität.

N. Gschwend - W. O. Frey

Die Herausgeber 